\title{
Crosslinked Polyurethane-Based Gels
}

\author{
Ha Phung,,${ }^{* \dagger}$ Etienne Schacht, ${ }^{*, * *}$ Filip Du Prez, ${ }^{*}$ Jan Gelan,,${ }^{* * *}$ \\ Peter AdRIAESENS, ${ }^{* * *}$ and Liesbet STORME*** \\ *Department of Organic Chemistry, University of Ghent, 281, S4-bis Krijgslaan, \\ 9000 Ghent, Belgium \\ ** Institute of Biomedical Technology, University of Ghent, Belgium \\ ${ }^{* * *}$ Limburg University, Institute for Materials Research (IMO), Department SBG, Universitaire Campus, \\ Building D, B-3590 Diepenbeck, Belgium
}

(Received March 20, 2002; Accepted February 3, 2003)

\begin{abstract}
Crosslinked polyurethanes (CPUs) were synthesized by reaction of (ethylene oxide-propyleneoxideethyleneoxide) (EO-PO-EO) triblock copolymer with diisocyanate and a low molecular weight triol as a crosslinking agent. The short diol was used as a chain extender. The effect of some factors such as the nature and content of PAOs, short diol chain extender and triol crosslinking agent on the phase separation of CPUs was studied by infrared spectroscopy (IR) and differential scanning calorimetry (DSC). The presence of amine group-containing compounds favors the phase separation of CPUs. The swelling behavior of CPUs in the different media such as aqueous solution and ethanol (EtOH)-water mixture has been investigated. The CPUs show the negatively thermosensitive swelling behavior. The collapsing swelling process in response to the change in environmental temperature was observed in CPUs prepared from EO-PO-EO triblock copolymer having a commercial code L92. In a EtOH-water mixture, CPUs reached the maximum swelling degree at the ratio $\mathrm{EtOH} /$ water $\cong 80 / 20$ at the whole range of the environmental temperature due to the coupling effect.
\end{abstract}

KEY WORDS Gels / Crosslinked Polyurethane / Phase Separation / Swelling Behavior / Coupling Effect /

Interest in polyurethane (PU) generally for the industry and in particular for the biomedical application stems largely from their properties such as the excellent mechanical properties, resistance to biodegradation and favorable biocompatibility. ${ }^{1,2}$ Segmented thermoplastic PU has been extensively studied. However, the investigation on crosslinked PU (CPU) has been still limited. (Ethylene oxide-propyleneoxide-ethyleneoxide) triblock copolymers (EO-PO-EO copolymers) prove to be useful polymer because of their anomalous solubility pattern, low toxicity, nonirritating nature and biocompatibility. ${ }^{3}$ The hydrophilicity of hydrogels prepared from EO-PO-EO copolymer is easily varied by using different content ratio of EO unit over PO unit. CPU has been prepared by crosslinking reaction of macrodiol with diisocyanate $\left(4,4^{\prime}\right.$-dicyclohexylmethanediisocyanate) in presence of a small amount of 1,2,6-hexanetriol (HXT) or triethanolamine (TEA) as a crosslinking agent. The various content and type of short diol such as 1,4butanediol (BD) or $N$-methyldiethanolamine (MDEA) were used as a chain extender.

The aim of this article is to investigate the composition-morphology relationship of CPUs and the swelling behavior of CPUs in different media. The swelling kinetic of CPUs in aqueous solutions can be studied by two experimental techniques. Firstly, the mass change is followed by weight gain measurement. Secondly, the time-dependent concentration profile of the increased solvent is followed by magnetic resonance imaging (MRI). MRI has been demonstrated as a powerful alternative technique., ${ }^{4}$ When the diffusion of liquid into CPUs is followed by MRI, the time dependence of the distance diffused can be expressed as below:

$$
\log \text { (distance) } \sim n \times \log \text { (time) }
$$

The value $n$, sometime it is called as a monitoring index, defines four possible situations and characterizes the type of transport as below:

i) For a perfectly Fickian process, where the rate of diffusion of liquid is much less than that of the polymer segmental relaxation rates, $n=0.5$ (Fickian or transport case I).

ii) When the penetrant velocity is far greater than the chain stretching rate. The rate of swelling is zero-order kinetic and $n=1$ (transport of case II).

iii) When the rate of diffusion and segmental relaxation are comparable, the value of $n$ falls between 0.5 and 1 (the abnormal transport).

iv) When $n>1$ : the process is called as a transport of super case II.

${ }^{\dagger}$ Present address: Department of Science and Technology, Ministry of Industry, 54 Hai Ba Trung Street, Hanoi, Vietnam. 
Table I. Characteristics of macrodiols

\begin{tabular}{cccccc}
\hline Macrodiolss & L92 & P84 & P75 & PEG 400 & PEG 2000 \\
\hline${\mathrm{EO} / \mathrm{PO}^{\mathrm{a}}(\mathrm{mol} / \mathrm{mol})}$ & $4.7 / 10$ & $10.7 / 10$ & $15.2 / 10$ & $100 / 0$ & $100 / 0$ \\
$\mathrm{I}_{\mathrm{OH}}{ }^{\mathrm{b}}$ & 40.4 & 35.7 & 37.9 & 228.3 & 50.9 \\
$M_{\mathrm{n}}$ & $2780^{\mathrm{c}}$ & $3140^{\mathrm{c}}$ & $2960^{\mathrm{c}}$ & $400^{\mathrm{d}}$ & $2000^{\mathrm{d}}$ \\
\hline
\end{tabular}

a Determination by ${ }^{1} \mathrm{H}$ NMR. ${ }^{b}$ Hydroxyl number was determined by esterification with acetic anhydride and titrimetric analysis of the carboxylic acid group. ${ }^{\mathrm{c}}$ The molecular weight of macrodiols was determined from the hydroxyl number. ${ }^{\mathrm{d}}$ Data from supplier.

\section{EXPERIMENTAL}

\section{Materials}

Poly(oxyethylene)glycol (PEG2000, $\left.M_{\mathrm{n}}=2000\right)$ and PEG400 $\left(M_{\mathrm{n}}=400\right)$ were obtained from Aldrich (Bornem, Belgium). The EO-PO-EO copolymer with a commercial code such as L92, P84, and P75 were a kind gift of ICI (Everberge, Belgium). The water trace of poly(oxyethylene)glycol and EO-PO-EO copolymers (both are called as macrodiols) has been removed by azeotropic distillation with toluene. The molecular weight, the hydroxyl number and the ratio EO content over PO of the starting PAOs are characterized in Table I. All reagents such TEA, HXT, BD and MDEA were purchased from Aldrich. 4,4'-Methylene bis(cyclohexyl isocyanate) was obtained by Bayer and was dried before use.

\section{Equipment}

FT-IR spectrum was recorded on a PerkinElmer spectrophotometer model 1600 FT-IR. The thermal transition temperatures of CPUs were recorded by calorimeter PerkinElmer DSC 7 at heating rate of $10{ }^{\circ} \mathrm{C} \mathrm{min}^{-1}$. ${ }^{1} \mathrm{H}$ nuclear magnetic resonance (NMR) spectra were obtained at $360 \mathrm{MHz}$ with a Burker Spectrometer.

\section{Synthesis of CPUs}

Although the composition of the CPUs was changed, they all comply with the following equimolarity:

$$
\begin{aligned}
& {[\mathrm{OH}] \text { of macrodiol }+[\mathrm{OH}] \text { of short diol }} \\
& +[\mathrm{OH}] \text { of triol }=[\mathrm{NCO}] \text { of diisocyanate. }
\end{aligned}
$$

Nomenclature is based on the crosslinker, macrodiol, short diol, and their amount. One of samples is coded as illustrated in the following sample: CT-L92-1.01MDEA. $\mathrm{C}$ refers to the crosslinked PU. T refers to the type of crosslinker (T: TEA to distinguish from $\mathrm{H}$ : HXT). L92 refers to the macrodiol. The number 1.0 notes the amount of crosslinker in mole. Last letter MDEA represents the kind of short diol. The number 1 indicates the amount of short diol in mole.

\section{Swelling Kinetic Evaluated by MRI Measurement}

The cylindrical-shaped specimens with a diameter of $10 \mathrm{~mm}$ are exposed to water in test tubes. The ingress of water in CPUs was followed by chemical shift selectively. Since the solvents were continuously surrounding the polymer samples, the diffusion process was never interrupted.

\section{Equilibrium Swelling Degree in Aqueous Solutions}

The equilibrium swelling degree of CPUs in aqueous solutions was calculated by eq 2 :

$$
W(\%)=\left[\left(W_{\mathrm{h}}-W_{\mathrm{d}}\right) / W_{\mathrm{d}}\right] \times 100 \%
$$

Where $W_{\mathrm{h}}$ and $W_{\mathrm{d}}$ are the weight of hydrated and dry gel, respectively.

The particular swelling experiment was designed to investigate the swelling behavior of CPUs in a EtOHwater mixture at different temperatures via the total equilibrium swelling degree and selective swelling absorption of gels. The swelling degree of CPUs in a $\mathrm{EtOH}-$ water mixture is calculated as in eq 3:

$$
S_{\mathrm{w}}=100 \% \times\left(W_{\mathrm{h}}-W_{\mathrm{d}}\right) / W_{\mathrm{d}} \times \delta
$$

With $\delta$ : gravimetric density of the EtOH-water mixture.

The sorption selectivity of CPUs swollen in a EtOHwater mixture was determined by gas chromatography (GC) analysis. To do that, 3-4 g of CPUs were swollen to reach the equilibrium. Then swollen gel was immersed in $2 \times 50 \mathrm{~mL}$ of methanol to extract the solvent mixture in the gel. Ethanol content in the membrane before and after extraction was determined by GC, type HP6890, GP-MSD, Hewlett Packard, Mass Selection Detector.

\section{RESULTS AND DISCUSSION}

\section{Phase Separation of CPUs}

The importance of microphase separation of polymer network in controlling their properties leads to the effort to characterize the degree of microphase separation. The soft segment of CPU is formed in a reaction of macrodiol with diisocyanate. The hard segment of $\mathrm{CPU}$ is formed in a reaction of triol and short diol with 
diisocyanate. The characterization of the microdomain of CPU was studied by IR and DSC technique. Various techniques such as thermal analysis, ${ }^{6}$ dynamic mechanical analysis, ${ }^{7}$ and scanning technique ${ }^{8}$ have been used to investigate the morphology and hydrogen bonding of thermoplastic PU. Vibrational spectroscopy is one of the principle techniques for examining chain-chain interaction and conformational order in polymer., ${ }^{9} 10$ The hydrogen bonded stretching band is shifted to lower frequencies, broadened and intensified several fold. In CPU, both intra-urethane (within the hard segment) and inter-urethane (between the hard and soft segment) hydrogen bonding can occurs. Hydrogen bonds result from the mainly following interactions:

- Hydrogen atom of urethane $\mathrm{NH}$ group with urethane carbonyl group $(\mathrm{C}=\mathrm{O})$.

- Hydrogen atom of urethane $\mathrm{NH}$ group with ether oxygen

- Hydrogen atom of urethane NH group with another proton acceptor such as nitrogen atom in amine groupcontaining compounds (TEA, MDEA).

There is a competition between ether oxygen of PAO, carboxyl group and nitrogen atom to form a hydrogen bond with urethane $\mathrm{N}-\mathrm{H}$ group. This competition is a governing factor for the segregation of the hard segment and soft segment in CPUs. It is known from a numerous literatures ${ }^{11,12}$ that the absorption of hydrogen bonded urethane NH groups appears at 3300$3380 \mathrm{~cm}^{-1}$, the non-bonded NH groups gives an absorption band at $3450-3600 \mathrm{~cm}^{-1}$. The frequency absorption of hydrogen bonded carbonyl group is centered at about $1700 \mathrm{~cm}^{-1}$ and non hydrogen bonded carbonyl group is known to shift to the range 1740$1720 \mathrm{~cm}^{-1}$.

FT-IR spectra of PU crosslinked by TEA (CT-L921.0) and HXT (CH-L92-1.0) are shown in Figure 1. The urethane carbonyl absorption peak of CT-L92-1.0 splits into two peaks $\left(1692 \mathrm{~cm}^{-1}\right.$ : hydrogen bonded and $1701 \mathrm{~cm}^{-1}$ : non-hydrogen bonded) while this band of CH-L92-1.0 remained faintly hydrogen bonded. The urethane NH absorption band of CT-L92 is almost hydrogen bonded. It indicates that the urethane $\mathrm{NH}$ group of CT-L92-1.0 is attached to the urethane carbonyl group. In contrast, the peak of urethane $\mathrm{NH}$ group of CH-L92-1.0 splits into two bands $\left(3458 \mathrm{~cm}^{-1}\right.$ : non-hydrogen bonded, $3334 \mathrm{~cm}^{-1}$ : hydrogen bonded). The stronger interaction of the intra-urethane hydrogen bonded carbonyl group $(\mathrm{NH} . . \mathrm{O}=\mathrm{C})$ in the structure of CT-L92 results from the interaction of urethane $\mathrm{NH}$ and carbonyl group. The presence of the nitrogen atom in TEA probably contributes to the stronger intraurethane hydrogen bonding interaction, because on one hand TEA interacts with urethane $\mathrm{NH}$ group and on the
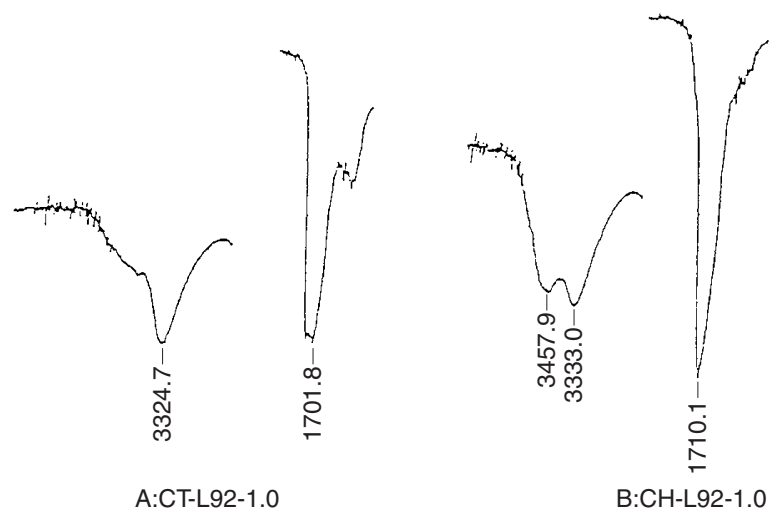

Figure 1. IR spectra of crosslinked polyurethane using different crosslinkers.

Table II. DSC data of some CPUs

\begin{tabular}{lcccccc}
\hline \multicolumn{1}{c}{ Samples } & $\begin{array}{c}T_{\mathrm{g}, \mathrm{s}} \\
\left({ }^{\circ} \mathrm{C}, \mathrm{I}\right)\end{array}$ & $\begin{array}{c}T_{\mathrm{g}, \mathrm{s}} \\
\left({ }^{\circ} \mathrm{C}, \mathrm{II}\right)\end{array}$ & $\begin{array}{c}T_{\mathrm{g}, \mathrm{h}} \\
\left({ }^{\circ} \mathrm{C}, \mathrm{I}\right)\end{array}$ & $\begin{array}{c}T_{\mathrm{g}, \mathrm{h}} \\
\left({ }^{\circ} \mathrm{C}, \mathrm{II}\right)\end{array}$ & $\begin{array}{c}T_{\mathrm{m}, \mathrm{s}} \\
\left({ }^{\circ} \mathrm{C}, \mathrm{I}\right)\end{array}$ & $w_{\mathrm{s}} / w_{\mathrm{h}}$ \\
\hline CT-L92-0.75 & -64.5 & -64.1 & 50.3 & - & - & $82 / 18$ \\
CT-L92-1.0 & -64.3 & $-64,0$ & 45.2 & - & - & $78 / 22$ \\
CT-L92-1.25 & -64.0 & -63.7 & 35.4 & - & - & $76 / 24$ \\
CT-L92-1.5 & -63.7 & -63.2 & 32.8 & - & - & $73 / 27$ \\
CT-L92-1.0-2BD & -63.2 & -63.5 & 31.4 & - & - & $66 / 34$ \\
CT-L92-1.0-3BD & -63.6 & -63.4 & 29.2 & - & - & $62 / 38$ \\
\hline
\end{tabular}

Where I: First heating; II: Second heating; s: soft segment; h: hard segment.

other hand it also promotes the interaction of urethane $\mathrm{NH}$ group with the urethane carbonyl group.

The glass transition temperature $\left(T_{\mathrm{g}}\right)$ of soft segment and the melting temperature $\left(T_{\mathrm{m}}\right)$ of CPUs prepared from L92 determined by DSC are presented in Table II. For all CPU samples prepared from L92, the soft segment was amorphous, since the thermal transition associated with the melting $\left(T_{\mathrm{m}}\right)$ of soft blocks could not be detected at any heating rate. The crystallization temperature was not also observed at any CPU sample. It comes from the fact that crosslinks in gels prevent the crystallization of the soft segment. Two distinct $T_{\mathrm{g}}^{\prime} \mathrm{s}$ corresponding to each segment were recorded in all DSC curves of CPUs having the different crosslinker content. The glass transition temperature of hard segment of CPUs prepared from L92 was observed only in the first heating. It is well known that the system consisted of two systems that have different $T_{\mathrm{g}}$ values is considered as a complete phase separated system if $T_{\mathrm{g}}$ of each component is independent each to other. As seen in Table II, the $T_{\mathrm{g}}$ of the hard segment is shifted gradually inward with the increased content of short diol and triol crosslinker. It indicated that one small portion of soft segment is solubilized in the hard segment. The glass transition behavior of CPUs consisted of different diol content shows the same trend as in the case of CPUs having the different crosslinker content. The shift inward of $T_{\mathrm{g}}$ of the hard segment, when the 


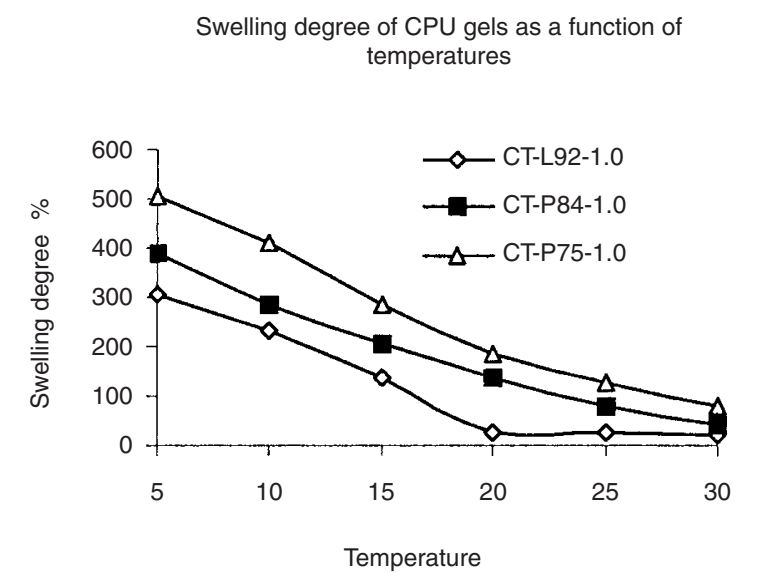

Figure 2. Swelling behavior of CPUs in aqueous solutions.

Table III. $n$ value calculated from MRI measurement

\begin{tabular}{ccc}
\hline Samples & \multicolumn{2}{c}{ Diffusion temperature } \\
\hline & $5{ }^{\circ} \mathrm{C}$ & $20{ }^{\circ} \mathrm{C}$ \\
CT-L92-1.0-1BD & 0.494 & 0.497 \\
CT-P84-1.0-1BD & 0.601 & 0.552 \\
\hline
\end{tabular}

short diol content is increased, indicates the less phase separation of CPUs.

\section{Swelling Behavior of CPUs in Aqueous Solutions}

The experimental program of this part was designed to prove the influence of temperatures on the swelling behavior and the discontinuous (collapsing) swelling behavior of some CPUs. The discontinuous temperature is defined as the inflection point on a plot describing the swelling degree as a function of temperatures.

All CPU samples showed the negatively temperature-dependent swelling behavior (see Figure 2). For most solutes, including polymers, the solubility in a given solvent increases with increasing temperature due to the enhanced compatibility of the chain and solvent. ${ }^{14}$ However a number of polymers, such as poly ( $N$-alkylacrylamide-co-methacrylic acid), ${ }^{15}$ poly (vinylmethylether) and $\mathrm{PEO}^{16,17}$ exhibit an inverse solubility-temperature behavior. The swelling degree of CPUs was increased in the following order: CPUs prepared from L92 < CPUs prepared from P84 < CPUs prepared from P75 due to the increase of EO/PO ratio. In the curve ${ }^{18}$ illustrating the swelling behavior of CPU prepared from L92, the collapsing point was observed at about $20^{\circ} \mathrm{C}$. The hydrophobic interaction or the hydrophilic-hydrophobic balance in hydrogels ${ }^{19}$ seems to be an important factor to explain the collapsing temperature-dependent swelling of hydrogels, since CPUs prepared from "pure" poly(oxyethylene)glycol such as PEG2000, PEG400 show only the continuous thermosensitive swelling behavior. ${ }^{18}$
Table IV. Diffusion coefficient $D_{\mathrm{f}}\left(\mathrm{cm}^{2} \mathrm{~s}^{-1}\right)$ calculated from MRI measurement

\begin{tabular}{ccc}
\hline Samples & \multicolumn{2}{c}{ Diffusion temperature } \\
\hline & $5{ }^{\circ} \mathrm{C}$ & $20^{\circ} \mathrm{C}$ \\
CT-L92-1.0-1BD & $1.54 \times 10^{-7}$ & $0.65 \times 10^{-7}$ \\
CT-P84-1.0-1BD & $9.45 \times 10^{-7}$ & $11.1 \times 10^{-7}$ \\
\hline
\end{tabular}

Table V. Ratio of EtOH/water in the CPU-based membrane

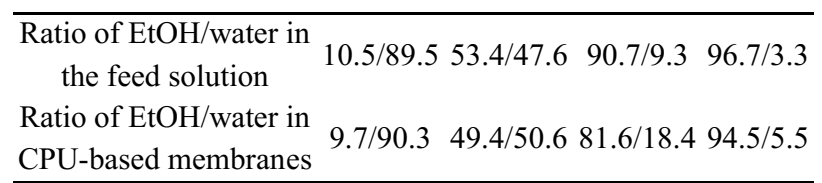

Swelling degree of CPUs as a function of water content in a mixture EtOH-water

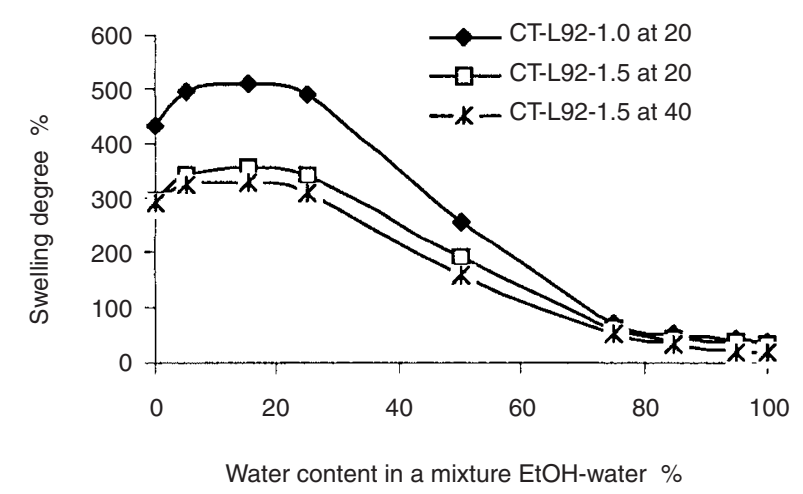

Figure 3. Swelling behavior of CPUs in a EtOH-water mixture.

\section{Swelling Kinetic Followed by MRI}

From the MRI measurement, the diffusion coefficient can be determined. The data illustrated in Table III demonstrated the Fickian process of water transport in CPUs prepared from L92 and P84 at the lower temperature and at room temperature. In Table IV the considerably lower diffusion coefficient $\left(D_{\mathrm{f}}\right)$ of CT-L92$1.0-1 \mathrm{BD}$ at $20^{\circ} \mathrm{C}$ comparing to that at $5^{\circ} \mathrm{C}$ was reported. The same phenomenon was not observed in CTP84-1.0-1BD. We can explain this difference by fact that CPUs prepared from L92 showed the collapsing swelling point at about $20^{\circ} \mathrm{C}$, while the swelling process of CPUs prepared from P84 is continuous. The diffusion coefficient of CPU prepared from P84 is higher than this prepared from L92 due to the higher swelling degree of CPUs prepared from P84.

\section{Equilibrium Swelling in a EtOH-Water Mixture}

The swelling behavior of gels was carried out via two factors: overall sorption and sorption selectivity. When gels are immersed in EtOH-water mixture, they will absorb both EtOH and water. Hence, $S_{\mathrm{w}}$ in the eq 3 consists of both EtOH and water.

The maximum swelling degree of samples at ratio 
$\mathrm{EtOH} /$ water changing from $80 / 20$ to $85 / 15$ is observed at the different temperatures (see Figure 3). This phenomenon can be explained in terms of so-called coupling effect that occurs due to strong interaction between solvent molecules of binary liquid mixtures of components, where the solubility or the diffusivity of a component is not only dependent on itself but also on the other components. ${ }^{20}$ Theoretically the sorption of small molecules and the orientation of the absorbed molecules change the configuration of the microvoid between the polymer chains and cause the facilitation or inhibition of absorption of the other component.

The maximum swelling degree in the mixture $\mathrm{EtOH} /$ water $\cong 80 / 20$ is not specific only for the gels containing both hydrophilic and hydrophobic component, but the same phenomenon is also observed in CPUs prepared from the pure PEOs. ${ }^{18}$ This experimental result was in total agreement with the result of some researchers. Lee $^{21}$ explained the maximal value at $\mathrm{EtOH} /$ water $\cong 80 / 20$ by the solubility parameter. $\mathrm{Du}$ Prez et al. ${ }^{22}$ observed similar finding for other gel based on polymethylmethacrylate crosslinked by $\alpha, \omega$ acrylate-terminated poly (1,3-dioxalane) and explained this phenomenon by the coupling effect. Semenova et $a l .{ }^{23}$ explained the maximum swelling degree of gels at $\mathrm{EtOH} /$ water $=80 / 20$ by coupling effect and also by increasing number of water layers on the active sites.

Sorption selectivity is defined as the composition of binary liquid mixture in gels. When immersed in the solvent mixture, gels can absorb preferentially one solvent over another one. By GC analysis, the ratio of $\mathrm{EtOH}$ and water in the feed mixture and in the membrane is illustrated in Table V. As a result, in each ratio of EtOH and water, EtOH and water do not interact with CPUs equivalently. The solvent composition in CPUs does not vary linearly with respect to the composition of the feed EtOH/water mixture. The gel always tends to prefer to absorb water than ethanol. According to $\mathrm{Neel}^{24}$ there are three main factors affected the preferential sorption:

- difference in molar volume between solvents having low molecular weight

- affinity towards the membrane

- solvents mutual affinity

Due to its small molar volume comparing to another organic solvents, water is preferentially absorbed. Generally the affinity between water and the organic solvent is low. In the case when water is one of components, water is always preferentially absorbed. The swelling degree of gels in a $\mathrm{EtOH}$-water mixture can change in respond to the ratio EtOH/water. Otherwise when immersed in a EtOH-water mixture, gels can absorb preferentially water over ethanol.

\section{CONCLUSIONS}

The IR and DSC data show that amine-containing CPUs (used TEA as a crosslinker, MDEA as a chain extender) favors the intra-urethane interaction when compared to non amine group-containing CPUs (used HXT as a crosslinker and $\mathrm{BD}$ as a chain extender). The interaction between NH-urethane group and the nitrogen atom in the tertiary amine group promotes the segregation between the hard and the soft segment. On the other hand, after forming, this intra-urethane hydrogen bonding interaction also enhances the intra-urethane hydrogen bonding interaction between $\mathrm{NH}$ urethane and carbonyl urethane group.

All CPUs prepared from L92 are amorphous due to the high crosslink density. Two distinct $T_{\mathrm{g}}^{\prime} \mathrm{s}$ were recorded on their DSC curves indicating the existence of two distinct regions, the hard and the soft, in CPUs prepared from L92. The inward shift of $T_{\mathrm{g}}$ of hard segment when the crosslinker content was increased, indicates the partial solubilization of hard segment in the soft segment domain.

The experimental result showed the negatively swelling thermosensitive behavior of CPUs in aqueous solutions. The swelling degree was decreased with increasing ratio $\mathrm{PO} / \mathrm{EO}$, short diol and crosslinker content. The results also showed the collapsing temperature-dependent swelling behavior of CPUs prepared from L92.

The swelling degree of gels exposed in EtOH-water mixture changes in response to the EtOH/water ratio and it reaches the maximum value at the ratio $\mathrm{EtOH} /$ water $\cong 80 / 20$ probably due to the coupling effect and the increase of number of water layers on the active sites. The results concerning the sorption swelling selectivity showed that in the mixture of $\mathrm{EtOH} /$ water, CPUs absorb preferentially water over ethanol due to the small volume of water, the low affinity of water to EtOH molecules and also due to the stronger affinity of water towards CPU-based membranes.

\section{REFERENCES}

1. R. Ratman and B. D. Ratner, J. Polym. Sci., Part A: Polym. Chem., 27, 2673 (1989).

2. K. Hayashi, H. Takano, T. Masuda, and M. Umezu, J. Biomed. Mater. Res., 19, 179 (1985).

3. "Ullman's Encyclopedia of Industrial Chemistry", WileyVCH, Ed., Weinheim, vol. A21, 579 (1992).

4. M. Ercken, P. Adriaensens, G. Reggers, R. Carleer, D. Vanderzande, and J. Gelan, Macromolecules, 29, 5671 (1996).

5. T. M. Hyde and L. F. Gladden, Polymer, 39, 811 (1998).

6. C. B. Wang and S. L. Cooper, Macromolecules, 16, 775 
(1983).

7. R. E. Camargo, C. W. Macosko, M. Tirell, and S. T. Wellinghoff, Polymer, 26, 1145 (1985).

8. C. M. Brunette, S. L. Hsu, M. Rossman, W. J. MacKnight, and N. S. Schneider, J. Polym. Eng. Sci., 21, 1837 (1981).

9. F. Papadimitrakopoulos, E. Sawa, and W. J. MacKnight, Macromolecules, 25, 4682 (1992).

10. K. Tashiro, K. Ono, Y. Minagawa, M. Kobayashi, T. Kawai, and K. Yoshino, J. Polym. Sci, Part B: Polym. Phys., 29, 1223 (1991).

11. J. L. Hong, C. P. Lillya, and J. C. W. Chen, Polymer, 33, 4347 (1991).

12. P. N. Lan, S. Corneillie, E. Schacht, M. Davies, and A. Shard, Biomaterials, 17, 2273 (1996).

13. D. J. Martin, G. F. Meijs, G. M. Renwick, P. A. Gunatillake, and S. J. McCarthy, J. Appl. Polym. Sci., 60, 557 (1996).

14. P. J. Flory, "Principle of Polymer Chemistry", Corneil University Press, Ithaca, N.Y., 1953.

15. A. S. Hoffman, A. Afrassiabi, and L. C. Dong, J. Controlled
Release, 4, 213 (1986).

16. M. van Boss and E. Schacht, Acta Pharm. Technol., 33, 120 (1986).

17. B. Gadner, R. Gurny, and E. Doelker, Drug Der. Ind. Pharm. 12 (11-13), 1613 (1986).

18. Phung Ha, Ph. D. Thesis in Chemistry, University of Ghent, Belgium 2000.

19. K. Suwa, K. Morishita, and M. Akashi, J. Polym. Sci., Part A: Polym. Chem., 35, 3087 (1997).

20. E. Drioli, S. Zhang, and H. Basile, J. Membr. Sci., 81, 43 (1993)

21. Y. K. Lee and S. C. Kim, Macromolecules, 19, 644 (1986)

22. F. E. Du Prez, E. J. Goethals, R. Schué, H. Qarouh, and F. Schué, Polym. Int., 46, 117 (1998).

23. S. I. Semenova, H. Ohya, and K. Soonatapa, Desalination, 110, 251 (1997).

24. J. Néel, in "Pervaporation membrane separation process", 2nd ed, R. Y. M. Huang, Ed., Elsevier Science Publishers B.V., Amsterdam, 1991. 